\title{
Editorial
}

\section{Final de Gestão}

Terminamos, com este número, nosso trabalho à frente da revista. Foram oito números, 145 artigos. Dentre eles, aproximadamente $40 \%$ foram de autores da região sudeste, $22 \%$, da região sul, $14 \%$, da região centro-oeste, $10 \%$, da região norte, $9 \%$, da região nordeste e $5 \%$, de universidades estrangeiras. A grande maioria envolveu dois ou três autores (49 e 21\%, respectivamente). Esse elevado número de artigos publicados foi possível graças ao trabalho conjunto das secretárias, do secretário de editoração, dos editores associados, dos diagramadores e das tradutoras. A todos, o meu sincero agradecimento pela seriedade, compromisso e competência no exercício de suas atividades. Em especial, agradeço ao secretário de editoração, Marcelo Bevenuti, pela contribuição inestimável na revisão final de cada artigo, uma contribuição que foi decisiva para a qualidade e pontualidade das publicações. Obviamente, a participação dos autores merece um reconhecimento à parte, não somente por terem escolhido nossa revista para a divulgação de seus trabalhos, mas também por terem colaborado com os editores com tanta presteza e boa vontade durante o processo de tramitação.

Encerramos nossa gestão com a certeza de termos feito o melhor possível Foi um trabalho árduo, mas os resultados foram reforçadores. Agora, uma nova equipe assumirá a responsabilidade das atividades da revista. Desejamos muito sucesso a todos!

Com muito pesar, relembro o falecimento, em maio passado, da professora Tereza Maria de Azevedo Pires Sério, da Pontifícia Universidade Católica de São Paulo. Tereza, ou Téia, como gostava de ser chamada, era uma professora muito admirada e querida por seus alunos, uma pesquisadora de prestígio inquestionável. Com seu vasto conhecimento da obra de Skinner e sua produção cientifica sobre metacontingências, variabilidade comportamental, operações estabelecedoras, dentre outros temas, muito contribuiu para o desenvolvimento da Análise do Comportamento no Brasil. A ela seremos eternamente gratos.

Este número, como já é prática editorial da revista, compreende artigos provenientes de diversas instituições espalhadas por todo o país, além de um artigo de universidade estrangeira, e referentes aos diversos saberes em psicologia. Acácia Aparecida Angeli dos Santos, Fermino Fernandes Sisto e Ana Paula Porto Noronha investigaram, com crianças, possíveis relações entre os testes TONI 3-Forma A e Cloze, os quais consistem, respectivamente, em medidas não-verbal e verbal de inteligência. O estudo de Aline Cardoso Siqueira e Débora Dalbosco Dell'Aglio identificou algumas variáveis individuais e familiares de crianças e jovens acolhidos em instituições governamentais e não-governamentais em decorrência de medidas de proteção judicial. Ana Maria Almeida Carvalho, Lúcia Vaz de Campos Moreira e Elaine Pedreira Rabinovich avaliaram quantitativamente as concepções de crianças, de diferentes regiões e níveis socioeconômicos diversos, acerca de suas famílias. Por sua vez, Luzia Iara Pfeifer, Yara Dias Martins e Jair Lício Ferreira Santos ana- lisaram a influência do gênero e do nível socioeconômico sobre as escolhas de atividades de lazer entre adolescentes.

Isabela Porpino Lemos e Eleonora Arnaud Pereira Ferreira identificaram comportamentos relevantes de crianças com diagnóstico de câncer e de seus acompanhantes e auxiliares de enfermagem, durante um procedimento de punção venosa para administração de quimioterapia. Alessandra Brunoro Motta e Sônia Regina Fiorim Enumo compararam os efeitos de uma intervenção psicológica lúdica centrada no problema com aqueles de uma intervenção caracterizada pelo brincar livre sobre o enfrentamento da hospitalização em crianças com câncer. No trabalho de Márcia Camaratta Anton e Cesar Augusto Piccinini foram averiguados os aspectos psicossociais de crianças e de suas famílias correlacionados com diferentes momentos do transplante hepático infantil. Ainda com relação a crianças, Daniela Aparecida Rissi Yamanaka, Roberto Benedito de Paiva e Silva, Maria de Lurdes Zanolli e Angélica Bronzatto de Paiva e Silva avaliaram as informações de pais de crianças surdas sobre o implante coclear, bem como suas expectativas sobre o futuro de seus filhos após o transplante.

Carmen Maria Bueno Neme e Marilda Emmanuel Novaes Lipp analisaram a ocorrência de estresse na história prévia de mulheres com e sem câncer, assim como o enfrentamento e a superação dessas situações estressantes. Maira Santos Marinho Moura, Karina Franco Zihlmann, Maria Lívia Tourinho Moretto, Newton Kara-José, Remo Susanna Junior e Mara Cristina Souza de Lucia investigaram o estresse em pacientes com glaucoma primário de ângulo aberto e a percepção desses pacientes acerca da doença e do tratamento. Joaquim C. Reis e Fernando S. Fradique identificaram níveis de desenvolvimento sociocognitivo nas concepções de adultos sobre saúde e doença.

Valéria Barbieri fez uma avaliação dos paradigmas qualitativo e quantitativo de investigação científica e comparou os fundamentos epistemológicos e metodológicos do Psicodiagnóstico Tradicional e Psicodiagnóstico Interventivo. Maurício da Silva Neubern discutiu a dor como uma experiência subjetiva do sujeito e a psicoterapia como um recurso capaz de modificar a influência do contexto relacionado à essa experiência. Fabio Scorsolini-Comin e Manoel Antônio dos Santos realizaram uma revisão da literatura no período de 1970 a 2008 com o objetivo de analisar o uso do construto satisfação conjugal. Morgana Scheffer, Graciela Gema Pasa e Rosa Maria Martins de Almeida verificaram a incidência de transtornos psiquiátricos em dependentes de álcool, cocaína e crack.

Ana Flávia de Oliveira Santos e Carmen Lúcia Cardoso investigaram a ocorrência de estresse, as estratégias de enfrentamento e a qualidade de vida de profissionais de saúde mental. Marina Campos Dessen e Maria das Graças Torres da Paz avaliaram a influência de configurações de poder e de características de personalidade sobre o bem-estar ocupacional de funcionários de empresas públicas e privadas. 
Teresa Cristina Martins Leite Imada, Marli Villela Mamede, Luiz de Souza e Raquel Gabrielli Biffi realizaram a adaptação e validação da Family Dynamics Measure II, uma escala a ser usada com familiares de mulheres com câncer de mama.

Sara Q. Matthiesen, preocupada com a qualidade das traduções para o português dos trabalhos de Reich, discutiu especificamente os termos utilizados nas traduções da pala- vra Trieb. Por fim, Fernando de Almeida Silveira e Richard Theisen Simanke analisaram o discurso dos sujeitos e objetos psicológicos segundo a ótica foucaultiana.

A leitura desses textos, com certeza, enriquecerá a todos nós.

Josele Abreu-Rodrigues

Editora

Conselho Editorial (2009 - 2011)

Ana Cleide Guedes Moreira (UFPA)

Ana Luíza Bustamante Smolka (UNICAMP)

Ana Raquel Rosas Torres (UCG e Uni-ANHANGUERA)

Carlos Barbosa Alves de Souza (UFPA)

Gardenia Abbad (UnB)

Gerson Américo Janczura (UnB)

Jair Lopes Junior (UNESP)

Jairo Eduardo Borges Andrade (UnB)

Lídia Natália Dobrianskyj Webwe (UFPR)

Lúcia Cavalcanti de Albuquerque Willian (UFSCar)

Luiz Augusto Monnerat Celes (UnB)

Maria Inês Gandolfo Conceição (UnB)

Raquel Maria Melo (UnB)

Silviane Bonaccorsi Barbato (UnB)

Tereza Cristina Cavalcanti Ferreira de Araújo (UnB)

Zeidi Araujo Trindade (UFES) 